\title{
USP5 Sustains the Proliferation of Glioblastoma Through Stabilization of CyclinD1
}

\author{
Gen $\mathrm{Li}^{1,2 \dagger}$, Tianquan $\mathrm{Yang}^{3 \dagger}$, Yanling Chen ${ }^{4+}$, Jianping $\mathrm{Bao}^{5 \dagger}$, $\mathrm{Di} \mathrm{Wu}^{1}$, Xiaohan $\mathrm{Hu}^{1}$, \\ Chenxi Feng ${ }^{1}$, Lixiao $X u^{1}$, Mei $L^{1}{ }^{1}$, Gang $L^{1}{ }^{1}$, Meifang Jin ${ }^{1}$, Yunyun $X u^{1}$, Rui Zhang ${ }^{6}$, \\ Guanghui Qian ${ }^{1 *}$ and Jian Pan ${ }^{1 *}$ \\ ${ }^{1}$ Institute of Pediatric Research, Children's Hospital of Soochow University, Suzhou, China, ${ }^{2}$ Laboratory of Molecular \\ Neuropathology, College of Pharmaceutical Sciences, Soochow University, Suzhou, China, ${ }^{3}$ Department of Neurosurgery, \\ Children's Hospital of Soochow University, Suzhou, China, ${ }^{4}$ School of Basic Medicine and Biological Sciences, Soochow \\ University, Suzhou, China, ${ }^{5}$ Department of Neonatology, Children's Hospital of Soochow University, Suzhou, China, ${ }^{6}$ Clinical \\ Pediatrics School, Soochow University, Suzhou, China
}

OPEN ACCESS

Edited by:

Cyril Corbet,

Fonds National de la Recherche Scientifique (FNRS), Belgium

Reviewed by:

Rachel Harding,

University of Toronto, Canada

Wenliang $\mathrm{Li}$,

University of Texas Health Science

Center at Houston, United States

*Correspondence:

Guanghui Qian

ghqian@suda.edu.cn

Jian Pan

panjian2008@163.com

${ }^{+}$These authors have contributed equally to this work

Specialty section: This article was submitted to Pharmacology of Anti-Cancer Drugs,

a section of the journal

Frontiers in Pharmacology

Received: 04 June 2021 Accepted: 04 August 2021 Published: 16 August 2021

Citation:

Li G, Yang T, Chen Y, Bao J, Wu D, Hu X, Feng $C, X u L$, Li M, Li G, Jin $M$,

Xu Y, Zhang R, Qian G and Pan J (2021) USP5 Sustains the Proliferation of Glioblastoma Through Stabilization of CyclinD1.

Front. Pharmacol. 12:720307. doi: 10.3389/fphar.2021.720307
Glioblastoma multiforme (GBM) is one of the most malignant primary tumors in humans. Despite standard therapeutic strategy with tumor resection combined with radiochemotherapy, the prognosis remains disappointed. Recently, deubiquitinating enzymes (DUBs) has been reported as potential cancer therapy targets due to their multifunctions involved in the regulation of tumorigenesis, cell cycle, apoptosis, and autophagy. In this study, we found that knockdown of ubiquitin specific protease (USP5), a family member of DUB, could significantly suppress GBM cell line U251 and DBTRG-05MG proliferation and colony formation by inducing cell cycle G1/S arrest, which was correlated with downregulation of CyclinD1 protein level. CyclinD1 had been reported to play a critical role in the tumorigenesis and development of GBM via regulating cell cycle transition. Overexpression of USP5 could significantly extend the half-life of CyclinD1, while knockdown of USP5 decreased the protein level of CyclinD1, which could be restored by proteasome inhibitor MG-132. Indeed, USP5 was found to directly interact with CyclinD1, and decrease its K48-linked polyubiquitination level. Furthermore, knockdown of USP5 in U251 cells remarkably inhibited tumor growth in vivo. Taken together, these findings demonstrate that USP5 plays a critical role in tumorigenesis and progression of GBM by stabilizing CyclinD1 protein. Targeting USP5 could be a potential therapeutic strategy for GBM.

Keywords: glioblastoma multiforme, USP5, CyclinD1, deubiquitination, cell cycle

\section{INTRODUCTION}

Glioblastoma multiforme (GBM) is one of the most malignant primary tumors originated by neuroglial stem or progenitor cells, which may occur at any age (Weller et al., 2015). There are approximately 13,000 new cases diagnosed in the USA every year (Reardon and Mitchell, 2017). Despite the development of current GBM therapeutic strategies includes surgical resection, chemotherapy, and radiotherapy, or a combination of these treatments, the prognosis remains gloomy (Ostrom et al., 2014). The average survival of GBM patients is about 14 months (Stupp et al., 2009), and the 5-years-survival rates are less than 9.8\% (Stupp et al., 2009; Carlsson et al., 2014). Hence, identification of new molecules involved in GBM tumorigenesis and progression is urgent for the development of more effective therapeutic strategies against this malignant tumor. 
Among post-translational modifications, ubiquitination plays a critical role by regulating a wide range of cellular biological progress, such as cell proliferation, cell cycle progression, apoptosis, inflammatory response, and DNA-damage repair (Hoeller and Dikic, 2009; Pal et al., 2014; Liu et al., 2017a; Yan et al., 2017). Ubiquitylation could be reversed by deubiquitinating enzymes (DUBs). DUBs are a large group of proteases which could mediate the removal of ubiquitin molecules from target proteins (Mevissen and Komander, 2017; Young et al., 2019). The human genome encodes approximately 115 DUBs, including six subclasses: ubiquitin specific proteases (USPs), ovarian tumor proteases (OTUs), ubiquitin carboxyterminal hydrolases (UCHs), MachadoJoseph disease proteases (MJDs), JAB1/MPN/Mov34 metalloenzymes (JAMMs), and motif interacting with Ubcontaining novel DUB family (MINDY) (Clague et al., 2013; Abdul Rehman et al., 2016; Weisberg et al., 2017). Recently, increasing investigations have suggested that USP family has critical function in tumor cell cycle (Kaistha et al., 2017), apoptosis (Wang et al., 2017), metastasis (Meng et al., 2019), and other biological progress (Ling et al., 2017; Gadotti and Zamponi, 2018; Sun et al., 2020).

In recent years, DUBs have exerted critical roles in GBM via targeting multiple key proteins involved in the regulation of tumorigenesis, cell cycle apoptosis, and autophagy. Previous studies had reported that USP1, USP8, USP11, and USP28 were indispensable for GBM growth via increasing the protein stability of ID1, FLIPS, PML, and c-Myc, respectively (Lee et al., 2010; Panner et al., 2010; Guo et al., 2013; Wu et al., 2014). Moreover, USP8, USP12, USP22 could promote the radioresistance of GBM stem cells by stabilizing Hedgehog pathways, Notch, and Sirt1, respectively (Chang et al., 2009; Wang et al., 2010; Santoni et al., 2013). Furthermore, USP22 promoted GBM chemoresistance to temozolomide by stabilizing ZEB1 (Siebzehnrubl et al., 2013). Thus, it would be of great importance to uncover more DUB members involved in GBM tumorigenesis and progression, which could be potential targets for GBM clinical therapy.

Ubiquitin specific protease 5 (USP5) belongs to the USP subfamily, which is located at chromosome $12 \mathrm{p} 13$, and encodes a 93.3 KDa protein (Wilkinson et al., 1995; AnsariLari et al., 1996). According to the current research, USP5 is reported to be involved in multiple biological progress, such as inflammatory response (Liu et al., 2018) and DNA damage repair (Nakajima et al., 2014). Furthermore, USP5 is also found to contribute to the tumorigenesis and progression of many malignancies, including neuroblastoma (Cheung et al., 2021), hepatocellular carcinoma (Liu et al., 2017b; Meng et al., 2019), multiple myeloma (Mollaoglu et al., 2017; Wu et al., 2020), pancreatic carcinoma (Kaistha et al., 2017; Li et al., 2017; Lian et al., 2020), ovarian cancer (Kim et al., 2018; Du et al., 2019), colorectal cancer (Xu et al., 2019), and non-small cell lung cancer (Ma et al., 2018; Xue et al., 2020). According to the GEPIA2 database (http://gepia2.cancer-pku.cn), compared with normal brain tissues, the mRNA level of USP5 in GBM tissues shows an increasing trend to some extent, indicating that USP5 may facilitate GBM tumorigenesis, and progression.

\section{MATERIALS AND METHODS}

\section{Cell Culture, Antibodies, and Chemicals}

The human GBM cell lines U251 and DBTRG-05MG, and human embryonic kidney cell line (293T) were purchased from the American Type Culture Collection. These cells were cultured in Dulbecco's modified Eagle's medium (DMEM) supplemented with $10 \%$ fetal bovine serum (FBS), $100 \mu \mathrm{g} / \mathrm{ml}$ of penicillin and 100 units/ $\mathrm{ml}$ of streptomycin. The primary antibodies against USP5 (ab154170), CyclinD1 (ab134175), CyclinE1 (ab33911), CDK2 (ab32147), CDK4 (ab108357), Ki67 (ab16667), PCNA (ab18197), Ubiquitin (linkage-specific K48, ab140601) were purchased from Abcam (USA), CDK6 (13,331), and $\beta$-Actin $(3,700)$ were purchased from Cell Signal Technology (USA). MG-132 (S2619) was purchased from Selleckchem (USA). Doxycycline hyclate (DOX, D9891) and Cycloheximide (CHX,239763) were purchased from Sigma (USA).

\section{Stable Cell Establishment for USP5 Overexpression}

Lentiviral plasmid expressing USP5 with $3 \times$ flag tag was generated by GENEWIZ (China). To generate lentiviral particles, HEK293T cells at $80 \%$ confluence in a $10 \mathrm{~cm}$ dish were co-transfected with $8 \mu \mathrm{g}$ target plasmid, $6 \mu \mathrm{g}$ psPAX2 (addgene, United States), $2 \mu \mathrm{g}$ pMD2.G (addgene, United States) using PEI (Sigma, United States) as a gene delivery carrier. After being washed and refreshed with the DMEM medium, cells were further cultured for $30 \mathrm{~h}$. The lentiviral particleenriched supernatant was harvested, filtered, and stored frozen at $-80^{\circ} \mathrm{C}$. After titration, these lentiviral particles were applied to infect U251 and DBTRG-05MG cells for $96 \mathrm{~h}$, and using $2 \mathrm{mg} / \mathrm{ml}$ puromycin to establish stable expression cells.

\section{Stable Cell Establishment for USP5 Knockdown}

U251 and DBTRG-05MG cell lines stably expressing USP5-specific shRNA or scrambled shRNA control were constructed and lentivirus particles were packaged by Genechem (China). U251 and DBTRG$05 \mathrm{MG}$ were infected with serial dilutions of lentiviral supernatant and selected for using $2 \mathrm{mg} / \mathrm{ml}$ puromycin for 2 weeks. The expression of shRNA should be induced by $2 \mathrm{ug} / \mathrm{mL}$ DOX for 3 days. The human USP5 shRNA targeting sequences are listed as follows. The targeting sequence for USP5-shRNA\# 1 was $5^{\prime}$ - TTGCCTTCATTAGTCACA T- $3^{\prime}$; the targeting sequence for USP5-shRNA\#2 was $5^{\prime}$ - TAGACA TGAACCAGCGGAT- $3^{\prime}$; the targeting sequence for USP5-shRNA\#3 was $5^{\prime}$ - CGAGGAGAAGTTTGAATTA-3'; the targeting sequences for scrambled shRNA was $5^{\prime}$ - TTCTCCGAACGTGTCACGT- ${ }^{\prime}$.

\section{Cell Proliferation and Colony Formation Assay}

Cell proliferation assays were performed by CCK- 8 assay. Cells $(2 \times$ $10^{3} /$ well) were seeded into 96 -well plates with $2 \mathrm{ug} / \mathrm{mL}$ DOX added into the cultural medium. $10 \mu \mathrm{L}$ CCK-8 solution (DOJINDO, Japan)/100 uL medium was added and incubated for an additional $2 \mathrm{~h}$. Then, the absorbance at $450 \mathrm{~nm}$ was measured using a Microplate Absorbance Reader (Bio-Rad, 
United States). As to colony formation assay, tumor cells $\left(1 \times 10^{3} /\right.$ well) were plated into 6 -well plates with $2 \mathrm{ug} / \mathrm{mL}$ DOX added into the cultural medium and incubated for 14 days. Cell colonies were fixed with $4 \%$ formaldehyde for $10 \mathrm{~min}$ and later stained with $0.1 \%$ crystal violet dye for $5 \mathrm{~min}$.

\section{Cell Migration Assay}

Cells were plated into 6-well dishes and treated with DOX for 3 days. Then cells were scratched in the center of the well and continuously cultured with $1 \%$ FBS medium. Wound images were photographed every $12 \mathrm{~h}$ using a light microscope (Nikon, Japan).

\section{Edu Staining Analysis}

Edu staining was performed using BeyoClick ${ }^{\mathrm{TM}}$ EdU Cell Proliferation Kit with Alexa Fluor 488 (Beyotime, China) according to the manufacturer's protocol. Briefly, cells were incubated with $10 \mu \mathrm{M}$ Edu for $2 \mathrm{~h}$ at $37^{\circ} \mathrm{C}$, and then fixed with $4 \%$ paraformaldehyde for $10 \mathrm{~min}$, and blocked with $3 \% \mathrm{BSA}$ in $0.1 \%$ PBS-Triton X-100 for another $1 \mathrm{~h}$. The cells were incubated with the Click Reaction Mixture for $30 \mathrm{~min}$ at room temperature in a dark place and then incubated with DAPI for $5 \mathrm{~min}$.

\section{Flow Cytometric Analysis}

Cell cycle and apoptosis were assayed using Cycle and Apoptosis Analysis Kit (Beyotime, China) according to the manufacturer's protocol. Briefly, cells were collected by centrifuging at $1,000 \mathrm{rpm}$ at $4^{\circ} \mathrm{C}$ for $5 \mathrm{~min}$, cell pellets were washed twice with cold PBS (Procell, China). For apoptosis, cells were labelled with propidium-iodide mixture and Annexin V-FITC and subsequently measured by the flow cytometer GALLIOS (Beckman Coulter, United States). For cell cycle, cells were resuspended and fixed with ice-cold ethanol (70\%) overnight, and then labelled with propidium-iodide mixture and subsequently measured by the flow cytometer GALLIOS. For data evaluation, the software FlowJo ver. 7.6.5 (Tree Star Inc., Oregon, United States) was used.

\section{RNA Extraction, cDNA Synthesis, and qRT-PCR}

Total RNA was extracted from tumor cells using TRIzol reagent (Invitrogen, United States), and cDNA was then synthesized with $5 \times$ all-in-one RT MasterMix (abm, Canada) according to the manufacturer's protocol. Quantitative real-time Reverse Transcription PCR (qRT-PCR) was conducted with $2 \times$ SYBR Green qPCR MasterMix (Bimake, United States). The relative mRNA expression was calculated after normalization to GAPDH. The primer sequences are as following: USP5, 5'CGGATTTGA CCTTAGCG-3' (Forward) and 5'-CTGCCATCGAAGTAGCG$3^{\prime}$ (Reverse), GAPDH, 5'-ATCATCCCTGCCTCTACTGG-3' (Forward) and $5^{\prime}$-CCCTCCGACGCCTGCTTCAC-3' (Reverse), CyclinD1, 5'-CCCTCGGTGTCCTACTTCA-3' (Forward) and $5^{\prime}$ - CTCCTCGCACTTCTGTTCCT- $3^{\prime}$ (Reverse), CyclinE1, 5' - CAGCCTTGGGACAATAATGC-3' (Forward) and $5^{\prime}$ - TTGCACGTTGAGTTTGGGTA $-3^{\prime}$ (Reverse), CDK2, 5' - CAGGATGTGACCAAGCCAGT-3' (Forward) and $5^{\prime}$ - TGAGTCCAAATAGCCCAAGG-3'
(Reverse), CDK4, 5'- ATGGCTACCTCTCGATATGAGC-3' (Forward) and $5^{\prime}$ - CATTGGGGACTCTCACACTCT-3' (Reverse), CDK6, 5'- GTGAACCAGCCCAAGATGAC-3' (Forward) and $5^{\prime}$ - TGGAGGAAGATGGAGAGCAC-3' (Reverse), PCNA, 5' - GGCGTGAACCTCACCAGTAT-3' (Forward) and $5^{\prime}$ - TTCTCCTGGTTTGGTGCTTC-3' (Reverse).

\section{Immunoblotting and Immunoprecipitation}

For protein extraction, cells were collected by centrifuging at $1,000 \mathrm{rpm}$ at $4^{\circ} \mathrm{C}$ for $5 \mathrm{~min}$, cell pellets were washed twice with cold PBS (Procell, China) and then re-suspended in appropriate RIPA lysis buffer (Beyotime, China). Protein concentration was detected by Enhanced BCA Protein Assay Kit (Beyotime, China). Western blotting was done by electrophoresing $20 \mu \mathrm{g}$ proteins on SDS-PAGE and subsequently transferring electrophoretically onto PVDF membranes (Millipore, Germany). Blocking was done in 5\% non-fat dry milk in $0.1 \%$ TBST for $1 \mathrm{~h}$ at room temperature and then incubated with appropriate primary at $4{ }^{\circ} \mathrm{C}$ overnight. Then the membranes were incubated with HRPlabeled corresponding secondary antibodies for $1 \mathrm{~h}$ at room temperature and Super Signal West Dura Extended kit (Thermo Scientific, United States) was used to detect the result. $\beta$-actin was used as the internal control.

For immunoprecipitation assay, the supernatants with respective antibodies were incubated on a rotor at $4^{\circ} \mathrm{C}$ overnight. Then the protein $\mathrm{G}$ agarose beads (Millipore, Germany) was added into the mixture and incubated with rotation for an additional $3 \mathrm{~h}$ at $4^{\circ} \mathrm{C}$. Next, the immunoprecipitates were washed three times with cold washing buffer. Finally, the immunoprecipitates resuspended in loading buffer containing $\beta$-mercaptoethanol were denatured by $100^{\circ} \mathrm{C}$ for $10 \mathrm{~min}$ and then were subjected to SDS-PAGE experiment.

\section{In Vivo Procedure for Intracranial GBM Cell Implantation}

All animal experiments were performed under the approval of the Animal Care and Use Committee at Children's Hospital of Soochow University, and the experimental procedures for all mice were performed in accordance with the Regulations for the Administration of Affairs Concerning Experimental Animals approved by the State Council of the People's Republic of China. (No. ESCU-201700047, ESCU-201700046).

Briefly, eight athymic nude mice were randomized into 2 groups, and anaesthetized with Ketalar and xylazine $(10 \mathrm{mg} / \mathrm{kg})$ and stuck into a stereotactic head frame. A 1.5$\mathrm{mm}$ bur hole was trained one metric linear unit anterior to the sutura on the proper hemisphere and a pair of metric linear unit lateral from the midplane. A Hamilton syringe fastened onto the pinnacle frame was injected $5 \times 10^{5} \mathrm{U} 251$ in $5 \mu \mathrm{L}$ PBS into the left striatum of the brain. The skin incision was then closed with 4-0 silk thread. Suitable medications were provided to scale back pain. To induce USP5 knockdown, mice were fed $2 \mathrm{mg} / \mathrm{ml}$ doxycycline in drinking water. The tumor tissues were collected after 30 days and prepared for IHC staining. The tumor volume was calculated as $\pi \mathrm{ls}^{2} /{ }^{6}$, where 1 represented the 


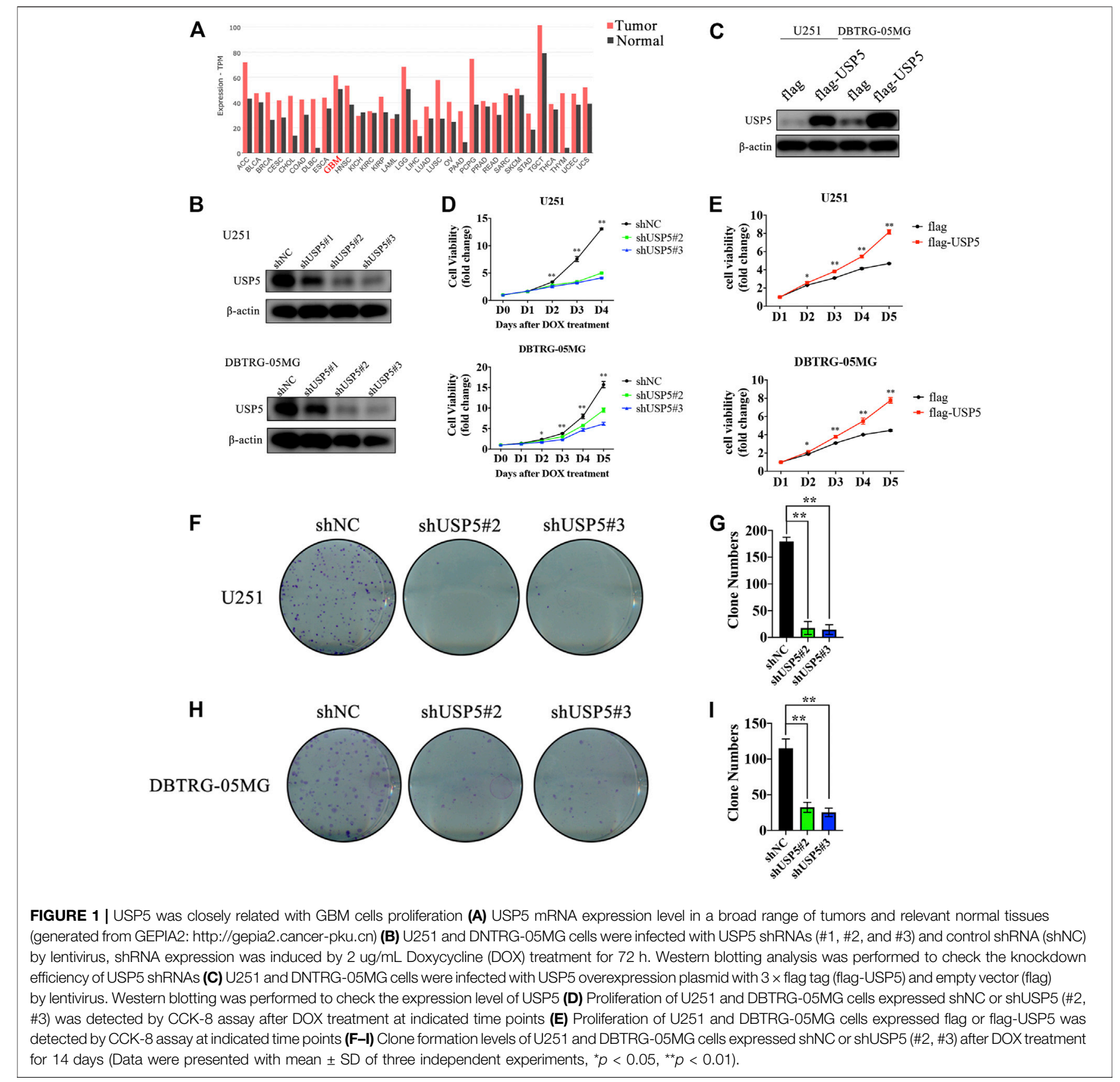

long side of the tumor, and s represented the short side of the tumor. For IHC staining, antigen retrieval was performed using $10 \mathrm{mM}$ citrate buffer ( $\mathrm{pH}$ 6.0) heated in a pressure cooker for $90 \mathrm{~s}$. USP5, CyclinD1, Ki-67, PCNA antibodies were applied overnight at $4^{\circ} \mathrm{C}$. Immunostaining was performed by using Envision + System and diaminobenzidine (DAB) visualization (Dako, Carpinteria, CA). Sections were counterstained with hematoxylin and examined by light microscopy.

\section{Statistics}

All data were displayed as means \pm standard deviations (SD). Statistical difference between the control and the experimental groups was analyzed by the two-tailed student's $t$-test. $p<0.05$ was deemed statistically significant.

\section{RESULTS}

\section{USP5 was Essential for GBM Cell Proliferation in vitro}

By searching the GEPIA2 database, USP5 was found to be highly expressed in most cancers, including GBM (Figure 1A). USP5 had been reported to contribute to the tumorigenesis and progression of many malignancies, however, the function of 

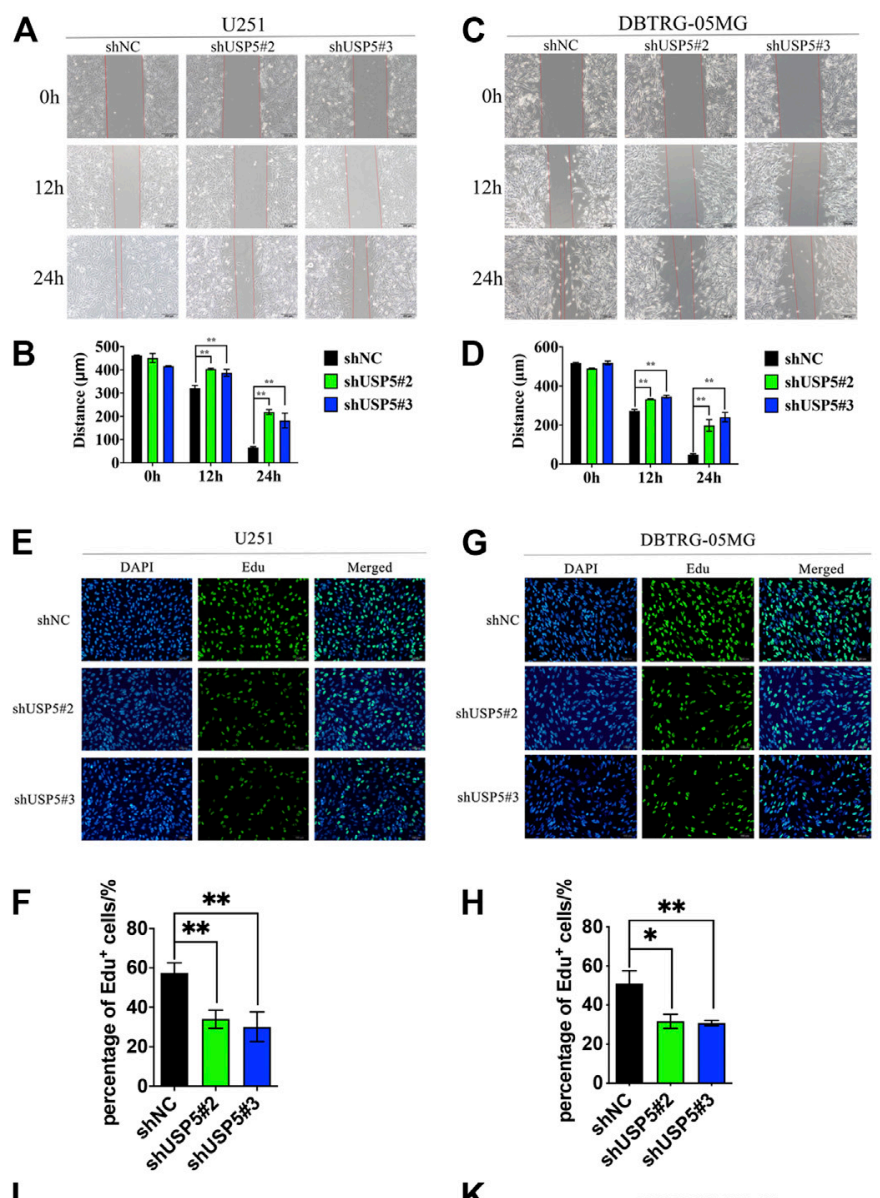

$\mathbf{H}$
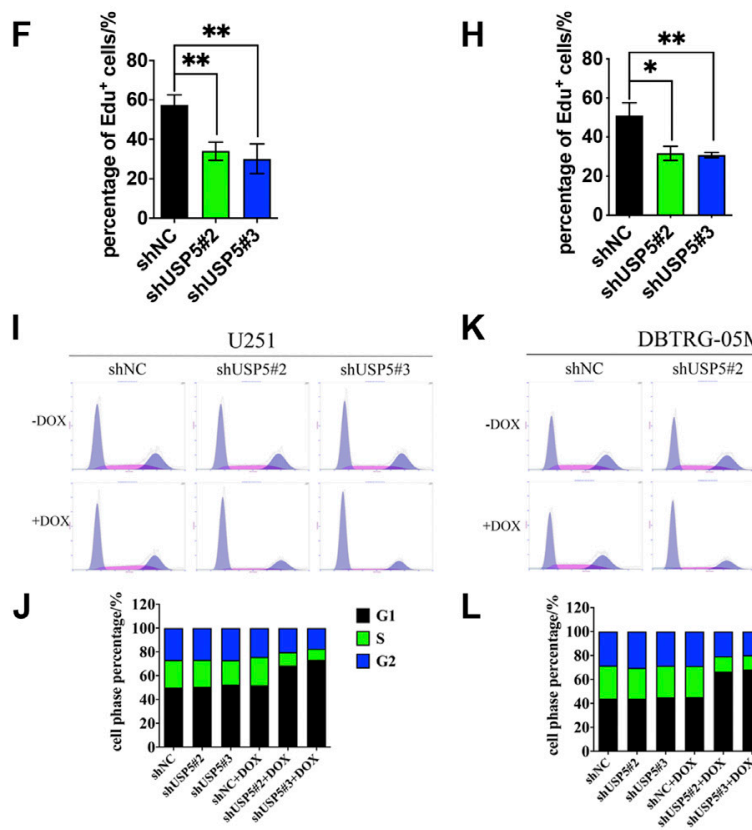

K
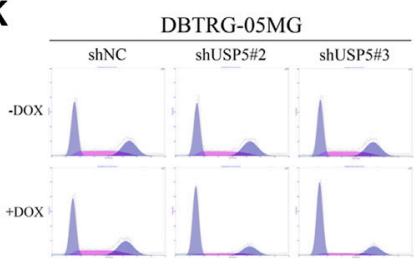

$\mathbf{L}$

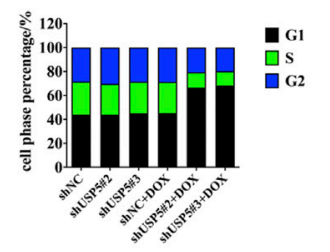

M

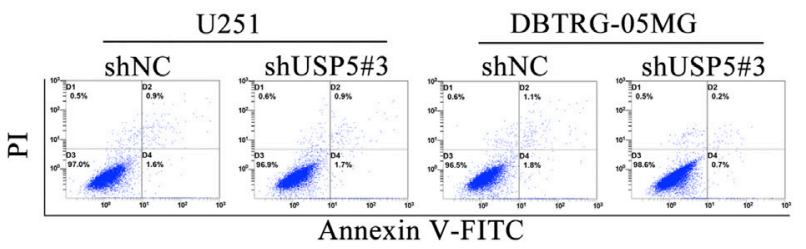

FIGURE 2 | USP5 knockdown inhibited GBM cell migration and induced cell cycle arrest in G1 phase (A-D) U251 and DBTRG-05MG cells stably expressed shNC or shUSP5 (\#2, \#3) were treated with $2 \mathrm{ug} / \mathrm{mL}$ DOX for $72 \mathrm{~h}$. Migration of U251 and DBTRG-05MG cells were determined based on a wound-healing assay. Black bar, $200 \mu \mathrm{m}$ (E-H) U251 and DBTRG-05MG cells stably expressed shNC or shUSP5 (\#2, \#3) were treated with $2 \mathrm{ug} / \mathrm{mL}$ Dox for $72 \mathrm{~h}$, then cells were incubated with $10 \mu \mathrm{M}$ Edu for $2 \mathrm{~h}$. Edu incorporation levels were determined by BeyoClick ${ }^{\mathrm{TM}}$ EdU Cell Proliferation Kit with Alexa Fluor 488. White bar, 100 um (I-L) U251 and DBTRG05MG cells stably expressed shNC or shUSP5 (\#2, \#3) were treated with $2 \mathrm{ug} / \mathrm{mL}$ DOX (+ DOX) or PBS (-DOX) for $72 \mathrm{~h}$. Cell cycle distribution of U251 and DBTRG05MG cells was assessed by PI staining and flow cytometry analysis. Representative graphs and statistical analysis of percentages at different cell cycle stages are shown (M) U251 and DBTRG-05MG cells stably expressed shNC or shUSP5 \#3 were treated with $2 \mathrm{ug} / \mathrm{mL}$ DOX (+ DOX) or PBS (-DOX) for 72 h. Cell apoptosis of U251 and DBTRG-05MG cells was assessed by PI and Annexin V-FITC staining and flow cytometry analysis (Data were presented with mean \pm SD of three independent experiments, $\left.{ }^{*} p<0.05,{ }^{\star *} p<0.01\right)$. 
A

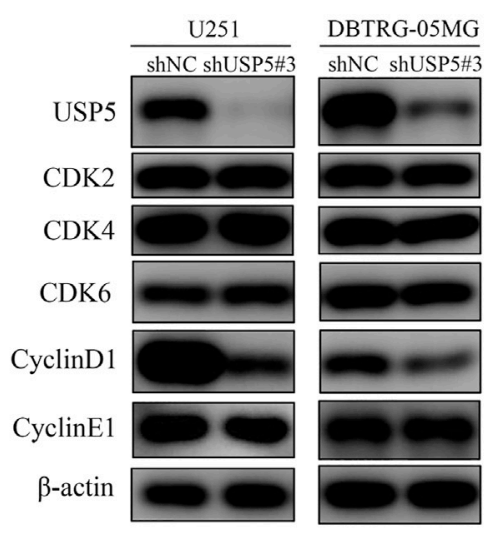

D

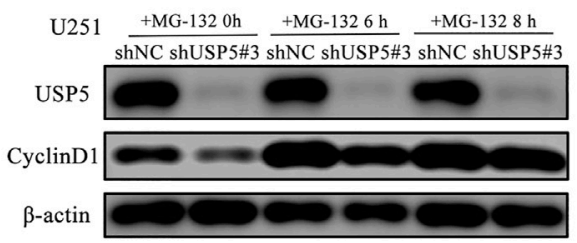

$\mathbf{F}$

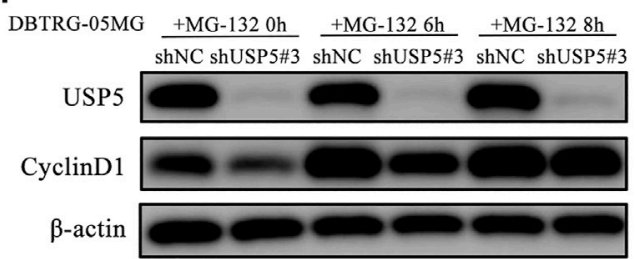

H

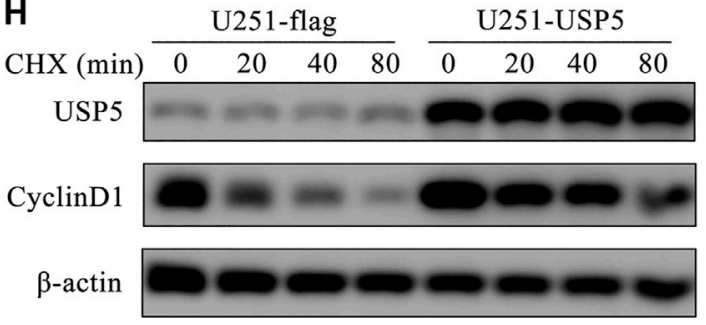

J DBTRG-05MG-flag DBTRG-05MG-USP5

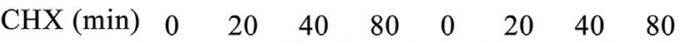

USP5

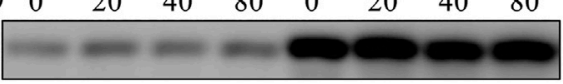

CyclinD1

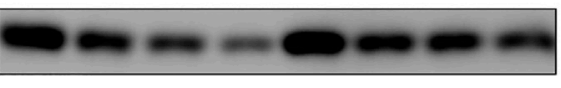

$\beta$-actin

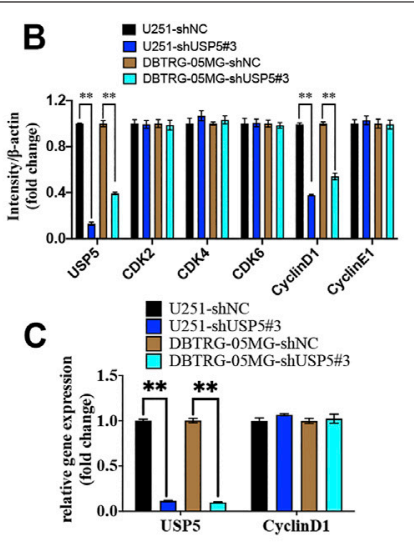

E

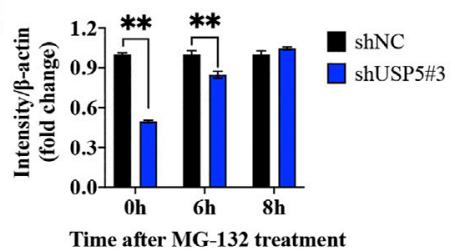

G

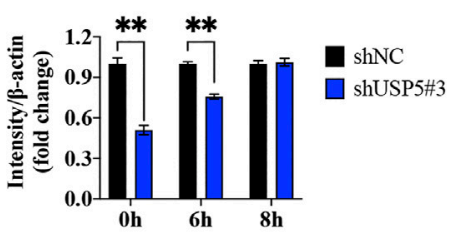

Time after MG-132 treatment

I

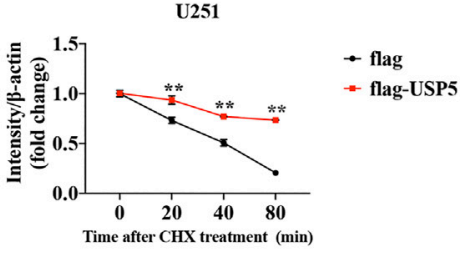

K

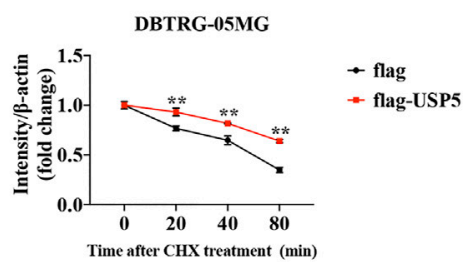

FIGURE 3 | USP5 maintained CyclinD1 stability (A, B) Western blotting analysis of USP5, CDK2, CDK4, CDK6, CyclinD1, CyclinE1, and $\beta$-actin protein levels in U251 and DBTRG-05MG cells stably expressed shNC or shUSP5\#3 treated with $2 \mathrm{ug} / \mathrm{mL}$ Dox for $72 \mathrm{~h}$ (C) Real-time PCR analysis of USP5 and CyclinD1 mRNA levels in U251 and DBTRG-05MG cells stably expressed shNC or shUSP5 \#3 treated with $2 \mathrm{ug} / \mathrm{mL}$ Dox for $72 \mathrm{~h}$ (D-G) U251 and DBTRG-05MG cells stably expressed shNC or shUSP5\#3 treated with $2 \mathrm{ug} / \mathrm{mL}$ Dox for $72 \mathrm{~h}$ were incubated with $20 \mathrm{MM}$ MG-132 for indicated time points. Western blotting analysis of USP5, CyclinD1, and $\beta$-actin protein levels (H-K) U251 and DBTRG-05MG cells stably expressed empty vector (flag) or USP5 overexpression (flag-USP5) were incubated with 100 $\mu \mathrm{g} / \mathrm{ml}$ $\mathrm{CHX}$ for indicated time points. Western blotting analysis of USP5, CyclinD1, and $\beta$-actin protein levels (Data were presented with mean \pm SD of three independent experiments, $\left.{ }^{\star} p<0.05,{ }^{\star \star} p<0.01\right)$. 
USP5 in GBM is still unknown. Thus, USP5 was knockdown or overexpressed in GBM cell lines U251 and DBTRG-05MG, respectively, via lentivirus-mediated plasmid transduction (Figures 2B,C). USP5 knockdown was induced by doxycycline treatment for $72 \mathrm{~h}$. The CCK8 assay showed that knockdown of USP5 significantly suppressed proliferation of U251 and DBTRG-05MG cells, while its overexpression relatively increased cell proliferation (Figures 1D,E). Moreover, USP5 knockdown also remarkably inhibited clone formation of U251 and DBTRG-05MG cells (Figures 1F-I). These results suggested that USP5 was required for GBM cell growth in vitro.

\section{Knockdown of USP5 Suppressed GBM Cell Migration and Cell Cycle Progression}

As USP5 could sustain GBM cell proliferation, leading us to further investigate its effect on cell phenotype. Wound healing assay showed that, compared with negative control, knockdown of USP5 could prominently prohibit U251 and DBTRG-05MG migration (Figures 2A-D). Moreover, Edu incorporation assay revealed that U251 and DBTRG-05MG were significantly arrested after USP5 knockdown (Figures 2E-H), indicating that USP5 was critical for GBM cell cycle progression. To confirm this hypothesis, cell cycle was determined by cytometry. As shown in Figures 2I-L, U251 and DBTRG$05 \mathrm{MG}$ were observably arrested in the $G_{1}$ phase after USP5 downregulation. Interestingly, cell apoptosis was not induced by USP5 knockdown (Figure $2 \mathbf{M}$ ). These results indicated that USP5 was important for GBM cell migration and cell cycle progression.

\section{USP5 Knockdown Decreased CyclinD1 Protein Stability}

The above studies showed that GBM cells were arrested in cell cycle G1 phase after USP5 knockdown, so we wondered whether USP5 regulated key proteins driving cell cycle G1 to $\mathrm{S}$ transition, including CDK2, CDK4, CDK6, CyclinD1, and CyclinE1 (Malumbres and Barbacid, 2009; Musgrove et al., 2011). As shown in Figures 3A,B, knockdown of USP5 in U251 and DBTRG-05MG cells specifically decreased CyclinD1 protein level. Interestingly, USP5 knockdown had no effect on CyclinD1 mRNA level (Figure 3C). USP5 is a member of deubiquitinases, which leading us to hypothesize the regulation of USP 5 on CyclinD1 protein stabilization. In U251 and DBTRG-05MG cells, the downregulation of CyclinD1 protein followed by USP5 knockdown could be rescued by treatment of proteasome inhibitor MG-132 (Figures 3D-G), which indicated that USP5 could prevent CyclinD1 from proteasome degradation. To confirm this finding, USP5 was overexpressed in U251 and DBTRG05MG cells, followed by treatment of CHX, an inhibitor of protein synthesis. The results showed that USP5 overexpression prolonged the half-life of CyclinD1 (Figures 3H-K). Taken together, these results suggested that USP5 sustained the stability of CyclinD1.

\section{USP5 Interacted With CyclinD1 Protein and Decreased Its k48-Linked Polyubiquitination Modification}

According to the above studies, CyclinD1 protein stability was dependent on USP5. Because the core function of USP5 is to prevent protein ubiquitination, and k48-linked polyubiquitination is the main type of modification for protein degradation, so we wondered whether USP5 removed k48-linked polyubiquitination chain from CyclinD1 protein. To confirm this supposition, co-immunoprecipitation assay was performed in U251 cells. As shown in Figures 4A,B, USP5 could interact with CyclinD1. To uncover the effect of USP5 on the ubiquitination level of CyclinD1, USP5 was overexpressed in U251 and DBTRG-05MG cells, respectively. The results showed that forced expression of USP5 noticeably decreased CyclinD1associated k48-linked polyubiquitination (Figure 3C). Overall, these results indicated that USP5 bound to CyclinD1 and downregulated the k48-linked polyubiquitination level.

\section{Knockdown of USP5 Inhibited GBM Growth in vivo}

To investigate the function of USP5 for GBM growth in vivo, we established orthotopic tumor models by intracranially implanting U251-shNC and U251-shUSP5\#3 cells into nude mice. As shown in Figures 5A,B, mice in the control group all developed tumors, while only three quarters in the USP5 knockdown group produced tumors. Moreover, compared with the control group, knockdown of USP5 remarkably decreased tumor sizes (Figures 5C,D). Immunohistochemical staining revealed that the levels of CyclinD1 and proliferation markers Ki-67 and PCNA were subsequently decreased after USP5 knockdown in xenograft sections (Figures 5E-G). These data indicated that USP5 was essential for GBM growth in vivo via regulating CyclinD1.

\section{DISCUSSION}

GBM remains one of the most malignant tumors worldwide with disappointed prognosis under current therapeutic strategies. Multiple molecules participated in the tumorigenesis and development of GBM. Hence, there is an urgent need to determine the vital genes concerned in tumor progress, providing promising strategies for precise therapy of patients with GBM. As ubiquitination modifications play a critical role in almost all cellular progresses, especially in cancers (Rape, 2018). Increasing evidences by recent studies indicate that DUBs are also involved in these activities (Harrigan et al., 2018). Our present investigation showed that USP5 was essential for GBM growth both in vitro and in vivo. We then confirmed the function of USP5 in the migration and cell cycle G1 to S progression of GBM cells. Furthermore, the underlying mechanism of USP5 in regulating GBM cell cycle arrest was clarified that USP5 directly targeted and stabilized CyclinD1 by suppressing its k48-linked polyubiquitination. These results indicated that USP5 could be an effective target for clinical GBM molecular therapy. 

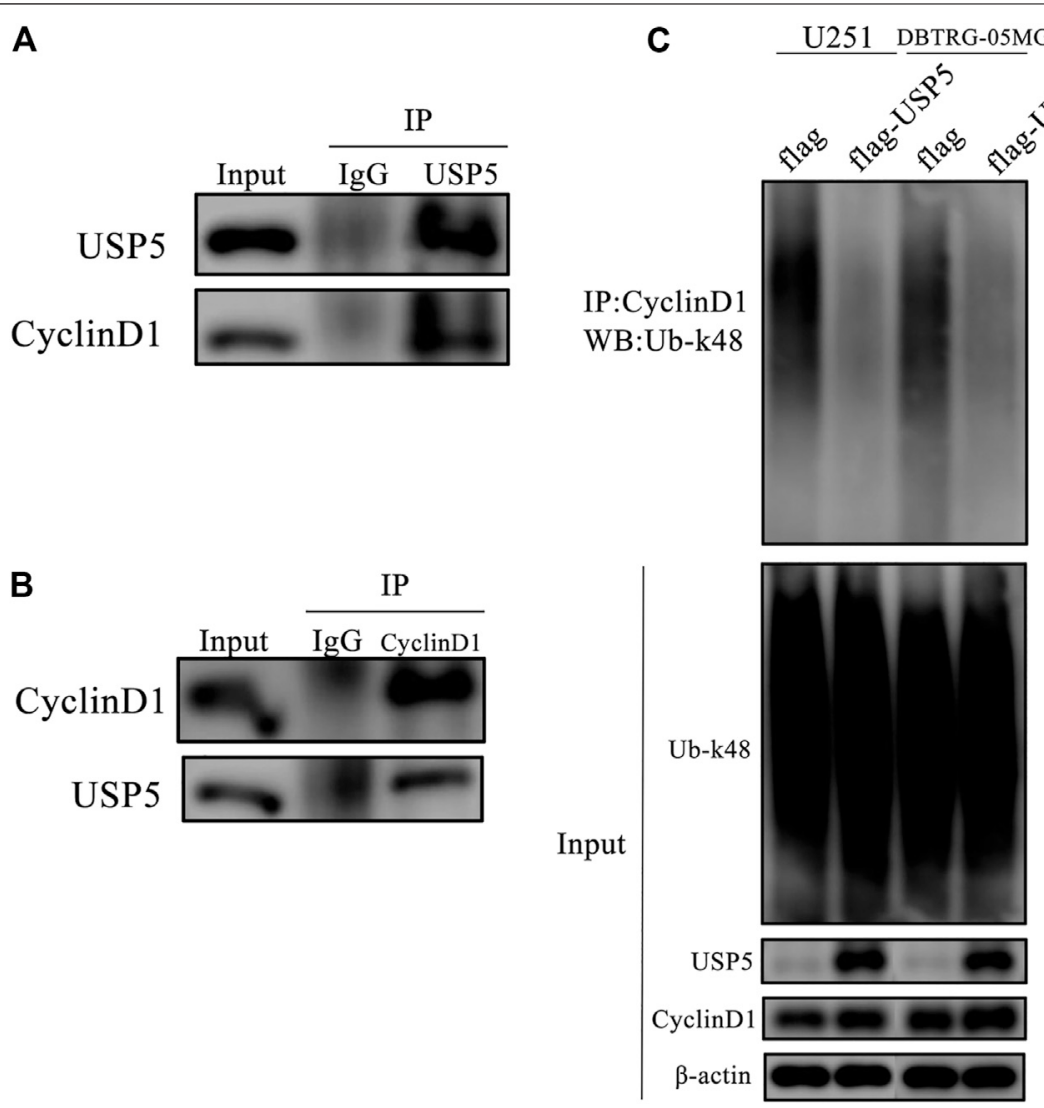

FIGURE 4 | USP5 directly interacted and deubiquitinated and CyclinD1 (A, B) USP5 (A) or CyclinD1 (B) was immunoprecipitated from U251 cells, respectively. Control immunoprecipitations were with nonspecific lgG, followed by western blotting of the precipitated proteins with antibodies for USP5 and CyclinD1 (C) CyclinD1 was immunoprecipitated from U251 and DBTRG-05MG cells stably expressed empty vector (flag) or USP5 overexpression (flag-USP5), respectively, followed by western blotting of the precipitated proteins with the antibody specifically for k48-linked ubiquitin (Ub-k48).

The proteins belonging to the USP family have already been reported to play vital roles in a variety of cancer associate progress. USP5 also has been shown to be a tumor suppressor in a number of malignant cancers by regulating different proteins. It had been reported that in GBM cell lines U87 and T98G, coknockdown of SF2/ASF1 in addition to USP5 inhibited cell proliferation and induced apoptosis via regulating hnRNPA1 through its deubiquitinase activity (Vashistha et al., 2020). Here, we reported that USP5 was also critical for GBM cell lines U251 and DBTRG-05MG proliferation via mediating cell cycle G1 to S progression without any alteration on cell apoptosis. These contrasts may be due to the tumor heterogeneity, as different GBM cell lines were used in the two independent investigations. Our data provided another insight into the functionality of USP5 in the tumorigenesis and development of USP5-related GBM. And the detailed mechanism of USP5 with different functions in GBM and how to identify the USP5-related GBM need to be further investigated.

In pancreatic carcinoma cells, knockdown of USP5 exhibited growth inhibition effects by suppressing cell cycle G1 to $\mathrm{S}$ transition, which was mediated by downregulating the cell cycle regulators (Kaistha et al., 2017). However, the underlying mechanism of USP5 in regulating cell cycle regulators is still under investigation.
Consistent with the previous studies, we found that knockdown of USP5 in GBM cell lines could significantly inhibit cell proliferation in vitro and in vivo via induced arrest of cell cycle $\mathrm{G} 1$ to $\mathrm{S}$ transition. Several proteins are critical for driving cell cycle G1 to $S$ transition, including CDK2, CDK4, CDK6, CyclinD1, and CyclinE1. Here, we revealed that among these proteins, knockdown of UP5 suppressed CyclinD1 protein level without any alteration of its mRNA level. This phenomenon is consistent with the regulation of USP5 as a deubiquitinase, which leading us to continue the following studies. Proteins modified with k48-linked polyubiquitination are subsequently degraded in proteasome (Swatek and Komander, 2016). In our study, decreased CyclinD1 protein caused by knockdown of USP5 could be rescued by treatment of proteasome inhibitor MG-132. Moreover, overexpression of USP5 obviously prolonged the half-life of CyclinD1 protein. Furthermore, USP5 was found to contact with CyclinD1 and decreased k48-linked polyubiquitination level on CyclinD1 protein. These results suggested that USP5 was essential for CyclinD1 protein stabilization.

It had been reported that USP 5 could be activated by multiple stimuli, such as heat stress (Xie et al., 2018) and nociceptive information (Stemkowski et al., 2017; Joksimovic et al., 2018). Previous study had revealed that USP5 was ubiquitinated and degraded by Smurf1 through the proteasome pathway (Qian 


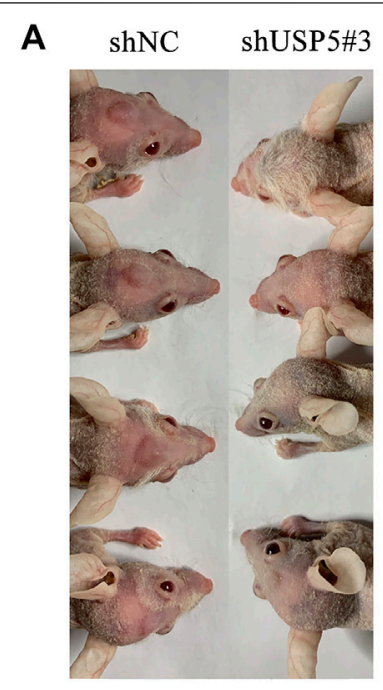

E

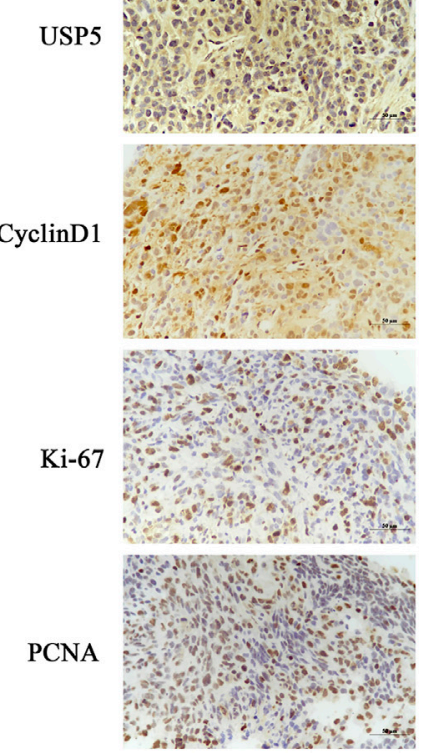

B

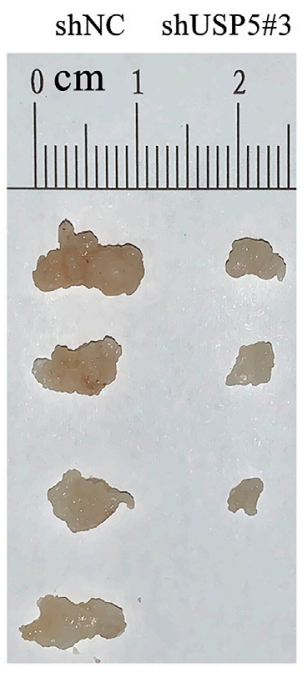

shUSP5\#3

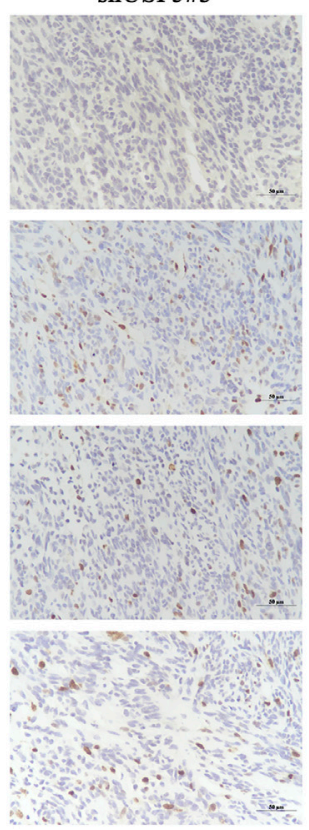

C

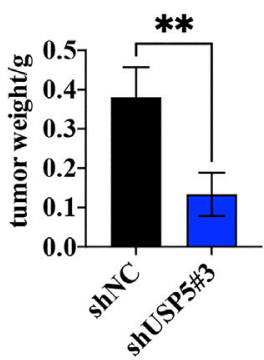

D

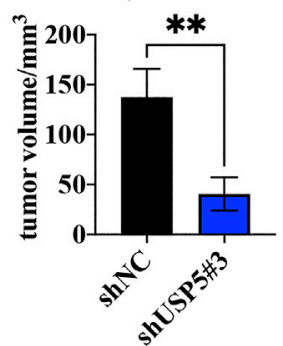

$\mathbf{F}$

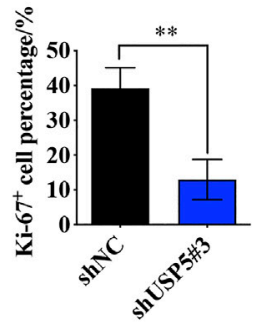

G

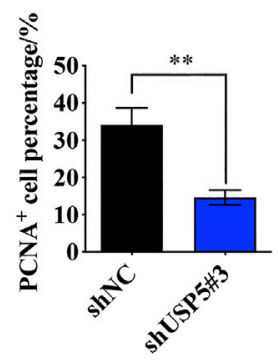

FIGURE 5 | Ablation of USP5 impaired the tumorigenicity of GBM cells in vivo. $5 \times 10^{5}$ U251 stably expressed shNC or shUSP5\#3 were intracranially injected into nude mice, respectively $(n=4)$. ShRNA expression was induced by $2 \mathrm{mg} / \mathrm{ml} \mathrm{DOX}$ in drinking water, and tumors were collected 30 days after induction (A, B) Photograph of xenograft tumors from shNC or shUSP5\#3 mice (C) Tumor weight from shNC or shUSP5\#3 mice (D) Tumor volume from shNC or shUSP5\#3 mice (F) IHC staining of USP5, CyclinD1, Ki-67, and PCNA in xenograft tumors from shNC or shUSP5\#3 mice (F, G) Ki-67 or PCNA positive cells count in IHC staining sections of xenograft tumors from shNC or shUSP5\#3 mice (Data were presented with mean \pm SD of three independent experiments, ${ }^{*} p<0.05,{ }^{* *} p<0.01$ ).

et al., 2016). Moreover, recent investigations had found that smurfl functioned as an oncoprotein via mediating PTEN ubiquitylation in GBM(Chang et al., 2018; Xia et al., 2020). Thus, further studies could focus on the mechanism of how USP5 is regulated in GBM.

A number of USPs play critical roles in different malignant tumors via regulating cell cycle progression (Kaistha et al., 2017; Kitamura and Hashimoto, 2021; Yuan et al., 2021) and are continuously investigated as potential therapeutic targets. Recently, a variety of chemical entities were developed as
USPs inhibitors, such as Pimozide, GW7674, Trifluooerazine, Rottlerin for USP1(Chen et al., 2011), ML364, LCAHA, 6TG for USP2 (Davis et al., 2016; Magiera et al., 2017; Chuang et al., 2018), GEN-6640, GEN-6776, FT67, FT827 for USP7 (Kategaya et al., 2017; Turnbull et al., 2017). USP14 inhibitor VLX1570 was the first DUB inhibitor to enter clinical trials for multiple myeloma therapy, but was deferred due to pulmonary toxicity (Wang et al., 2015; Rowinsky et al., 2020). Excitingly, increasing numbers of USP5 inhibitors were under drug discovery, such as RA-9 (Issaenko and Amerik, 2012), Vialimin A (Okada et al., 2013), 
Curcusone D (Cao et al., 2014), and showed a broad prospect for molecular therapy.

Taken together, our findings revealed a novel critical role for USP5 in the maintenance of GBM proliferation via deubiquitinated and stabilized CyclinD1 to promote cell cycle progression. These results provided another potent molecular target for GBM clinical therapy, and further research could screen or develop clinically available chemicals for USP5 inhibition.

\section{DATA AVAILABILITY STATEMENT}

The original contributions presented in the study are included in the article/supplementary material, further inquiries can be directed to the corresponding authors.

\section{ETHICS STATEMENT}

The animal study was reviewed and approved by the Animal Care and Use Committee at Children's Hospital of Soochow University.

\section{REFERENCES}

Abdul Rehman, S. A., Kristariyanto, Y. A., Choi, S. Y., Nkosi, P. J., Weidlich, S., Labib, K., et al. (2016). MINDY-1 Is a Member of an Evolutionarily Conserved and Structurally Distinct New Family of Deubiquitinating Enzymes. Mol. Cell 63, 146-155. doi:10.1016/j.molcel.2016.05.009

Ansari-Lari, M. A., Muzny, D. M., Lu, J., Lu, F., Lilley, C. E., Spanos, S., et al. (1996). A Gene-Rich Cluster between the CD4 and Triosephosphate Isomerase Genes at Human Chromosome 12p13. Genome Res. 6, 314-326. doi:10.1101/ gr.6.4.314

Cao, M.-N., Zhou, Y.-B., Gao, A.-H., Cao, J.-Y., Gao, L.-X., Sheng, L., et al. (2014). Curcusone D, a Novel Ubiquitin-Proteasome Pathway Inhibitor via ROSInduced DUB Inhibition, Is Synergistic with Bortezomib against Multiple Myeloma Cell Growth. Biochim. Biophys. Acta (Bba) - Gen. Subjects 1840, 2004-2013. doi:10.1016/j.bbagen.2014.02.006

Carlsson, S. K., Brothers, S. P., and Wahlestedt, C. (2014). Emerging Treatment Strategies for Glioblastoma Multiforme. EMBO Mol. Med. 6, 1359-1370. doi:10.15252/emmm.201302627

Chang, C.-J., Hsu, C.-C., Yung, M.-C., Chen, K.-Y., Tzao, C., Wu, W.-F., et al. (2009). Enhanced Radiosensitivity and Radiation-Induced Apoptosis in Glioma CD133-Positive Cells by Knockdown of SirT1 Expression. Biochem. Biophysical Res. Commun. 380, 236-242. doi:10.1016/ j.bbrc.2009.01.040

Chang, H., Zhang, J., Miao, Z., Ding, Y., Xu, X., Zhao, X., et al. (2018). Suppression of the Smurf1 Expression Inhibits Tumor Progression in Gliomas. Cell Mol Neurobiol 38, 421-430. doi:10.1007/s10571-017-0485-1

Chen, J., Dexheimer, T. S., Ai, Y., Liang, Q., Villamil, M. A., Inglese, J., et al. (2011). Selective and Cell-Active Inhibitors of the USP1/UAF1 Deubiquitinase Complex Reverse Cisplatin Resistance in Non-small Cell Lung Cancer Cells. Chem. Biol. 18, 1390-1400. doi:10.1016/j.chembiol.2011.08.014

Cheung, B. B., Kleynhans, A., Mittra, R., Kim, P. Y., Holien, J. K., Nagy, Z., et al. (2021). A Novel Combination Therapy Targeting Ubiquitin-specific Protease 5 in MYCN-Driven Neuroblastoma. Oncogene 40, 2367-2381. doi:10.1038/ s41388-021-01712-w

Chuang, S. J., Cheng, S. C., Tang, H. C., Sun, C. Y., and Chou, C. Y. (2018). 6Thioguanine Is a Noncompetitive and Slow Binding Inhibitor of Human Deubiquitinating Protease USP2. Sci. Rep. 8, 3102. doi:10.1038/s41598-01821476-w

\section{AUTHOR CONTRIBUTIONS}

JP and GQ designed and guided the study; GeL, TY, YC, and JB performed most of the experiments; DW and XH helped data analysis; CF and LX participated in lentivirus preparation and transfection; $\mathrm{ML}$ and GaL participated in the in vitro experiments; MJ and YX helped establish GBM xenograft model; RZ participated in plasmids construction. All authors read and approved the final manuscript.

\section{FUNDING}

This work was supported by the grants from the National Natural Science Foundation (81702737, 81902972, 82072767, 81770145, 81971477, 81701596), Natural Science Foundation of Jiangsu Province BK20191175, Jiangsu Provincial Medical Young Talents (QNRC2016756), the Applied Foundational Research of Medical and Health Care of Suzhou City (SYS2019086), Suzhou Health Talent Training Project (YX, GSWS2020048), the project of Soochow Science and Technology Plan (XH, SYS201761).

Clague, M. J., Barsukov, I., Coulson, J. M., Liu, H., Rigden, D. J., and Urbé, S. (2013). Deubiquitylases from Genes to Organism. Physiol. Rev. 93, 1289-1315. doi:10.1152/physrev.00002.2013

Davis, M. I., Pragani, R., Fox, J. T., Shen, M., Parmar, K., Gaudiano, E. F., et al. (2016). Small Molecule Inhibition of the Ubiquitin-specific Protease USP2 Accelerates Cyclin D1 Degradation and Leads to Cell Cycle Arrest in Colorectal Cancer and Mantle Cell Lymphoma Models. J. Biol. Chem. 291, 24628-24640. doi:10.1074/jbc.m116.738567

Du, Y., Lin, J., Zhang, R., Yang, W., Quan, H., Zang, L., et al. (2019). Ubiquitin Specific Peptidase 5 Promotes Ovarian Cancer Cell Proliferation through Deubiquitinating HDAC2. Aging 11, 9778-9793. doi:10.18632/aging.102425

Gadotti, V. M., and Zamponi, G. W. (2018). Disrupting USP5/Cav3.2 Interactions Protects Female Mice from Mechanical Hypersensitivity during Peripheral Inflammation. Mol. Brain 11, 60. doi:10.1186/s13041-018-0405-4

Guo, P., Nie, Q., Lan, J., Ge, J., Qiu, Y., and Mao, Q. (2013). C-myc Negatively Controls the Tumor Suppressor PTEN by Upregulating miR-26a in Glioblastoma Multiforme Cells. Biochem. Biophysical Res. Commun. 441, 186-190. doi:10.1016/j.bbrc.2013.10.034

Harrigan, J. A., Jacq, X., Martin, N. M., and Jackson, S. P. (2018). Deubiquitylating Enzymes and Drug Discovery: Emerging Opportunities. Nat. Rev. Drug Discov. 17, 57-78. doi:10.1038/nrd.2017.152

Hoeller, D., and Dikic, I. (2009). Targeting the Ubiquitin System in Cancer Therapy. Nature 458, 438-444. doi:10.1038/nature07960

Issaenko, O. A., and Amerik, A. Y. (2012). Chalcone-based Small-Molecule Inhibitors Attenuate Malignant Phenotype via Targeting Deubiquitinating Enzymes. Cell Cycle 11, 1804-1817. doi:10.4161/cc.20174

Joksimovic, S. L., Joksimovic, S. M., Tesic, V., Garcia-Caballero, A., Feseha, S., Zamponi, G. W., et al. (2018). Selective Inhibition of CaV3.2 Channels Reverses Hyperexcitability of Peripheral Nociceptors and Alleviates Postsurgical Pain. Sci. Signal. 11, 1. doi:10.1126/scisignal.aao4425

Kaistha, B. P., Krattenmacher, A., Fredebohm, J., Schmidt, H., Behrens, D., Widder, M., et al. (2017). The Deubiquitinating Enzyme USP5 Promotes Pancreatic Cancer via Modulating Cell Cycle Regulators. Oncotarget 8, 66215-66225. doi:10.18632/oncotarget.19882

Kategaya, L., Di Lello, P., Rougé, L., Pastor, R., Clark, K. R., Drummond, J., et al. (2017). USP7 Small-Molecule Inhibitors Interfere with Ubiquitin Binding. Nature 550, 534-538. doi:10.1038/nature24006

Kim, S. Y., Kwon, S. K., Lee, S. Y., and Baek, K. H. (2018). Ubiquitin-specific Peptidase 5 and Ovarian Tumor Deubiquitinase 6A Are Differentially 
Expressed in P53+/+ and P53-/- HCT116 Cells. Int. J. Oncol. 52, 1705-1714. doi:10.3892/ijo.2018.4302

Kitamura, H., and Hashimoto, M. (2021). USP2-Related Cellular Signaling and Consequent Pathophysiological Outcomes. Int. J. Mol. Sci. 22, 1. doi:10.3390/ ijms22031209

Lee, K.-y., Yang, K., Cohn, M. A., Sikdar, N., D’Andrea, A. D., and Myung, K. (2010). Human ELG1 Regulates the Level of Ubiquitinated Proliferating Cell Nuclear Antigen (PCNA) through its Interactions with PCNA and USP1. J. Biol. Chem. 285, 10362-10369. doi:10.1074/jbc.m109.092544

Li, X.-Y., Wu, H.-Y., Mao, X.-F., Jiang, L.-X., and Wang, Y.-X. (2017). USP5 Promotes Tumorigenesis and Progression of Pancreatic Cancer by Stabilizing FoxM1 Protein. Biochem. Biophysical Res. Commun. 492, 48-54. doi:10.1016/ j.bbrc.2017.08.040

Lian, J., Liu, C., Guan, X., Wang, B., Yao, Y., Su, D., et al. (2020). Ubiquitin Specific Peptidase 5 Enhances STAT3 Signaling and Promotes Migration and Invasion in Pancreatic Cancer. J. Cancer 11, 6802-6811. doi:10.7150/jca.48536

Ling, X., Huang, Q., Xu, Y., Jin, Y., Feng, Y., Shi, W., et al. (2017). The Deubiquitinating Enzyme Usp5 Regulates Notch and RTK Signaling duringDrosophilaeye Development. FEBS Lett. 591, 875-888. doi:10.1002/ 1873-3468.12580

Liu, J., Zhang, C., Zhao, Y., Yue, X., Wu, H., Huang, S., et al. (2017). Parkin Targets HIF-1alpha for Ubiquitination and Degradation to Inhibit Breast Tumor Progression. Nat. Commun. 8, 1823. doi:10.1038/s41467-017-01947-w

Liu, Q., Wu, Y., Qin, Y., Hu, J., Xie, W., Qin, F. X.-F., et al. (2018). Broad and Diverse Mechanisms Used by Deubiquitinase Family Members in Regulating the Type I Interferon Signaling Pathway during Antiviral Responses. Sci. Adv. 4, eaar2824. doi:10.1126/sciadv.aar2824

Liu, Y., Wang, W. M., Zou, L. Y., Li, L., Feng, L., Pan, M. Z., et al. (2017). Ubiquitin Specific Peptidase 5 Mediates Histidine-Rich Protein Hpn Induced Cell Apoptosis in Hepatocellular Carcinoma through P14-P53 Signaling. Proteomics 17, 1. doi:10.1002/pmic.201600350

Ma, X., Qi, W., Pan, H., Yang, F., and Deng, J. (2018). Overexpression of USP5 Contributes to Tumorigenesis in Non-small Cell Lung Cancer via the Stabilization of $\beta$-catenin Protein. Am. J. Cancer Res. 8, 2284-2295.

Magiera, K., Tomala, M., Kubica, K., De Cesare, V., Trost, M., Zieba, B. J., et al. (2017). Lithocholic Acid Hydroxyamide Destabilizes Cyclin D1 and Induces G 0/G 1 Arrest by Inhibiting Deubiquitinase USP2a. Cell Chem. Biol. 24, 458-470. doi:10.1016/j.chembiol.2017.03.002

Malumbres, M., and Barbacid, M. (2009). Cell Cycle, CDKs and Cancer: a Changing Paradigm. Nat. Rev. Cancer 9, 153-166. doi:10.1038/nrc2602

Meng, J., Ai, X., Lei, Y., Zhong, W., Qian, B., Qiao, K., et al. (2019). USP5 Promotes Epithelial-Mesenchymal Transition by Stabilizing SLUG in Hepatocellular Carcinoma. Theranostics 9, 573-587. doi:10.7150/thno.27654

Mevissen, T. E. T., and Komander, D. (2017). Mechanisms of Deubiquitinase Specificity and Regulation. Annu. Rev. Biochem. 86, 159-192. doi:10.1146/ annurev-biochem-061516-044916

Mollaoglu, G., Guthrie, M. R., Böhm, S., Brägelmann, J., Can, I., Ballieu, P. M., et al. (2017). MYC Drives Progression of Small Cell Lung Cancer to a Variant Neuroendocrine Subtype with Vulnerability to Aurora Kinase Inhibition. Cancer Cell 31, 270-285. doi:10.1016/j.ccell.2016.12.005

Musgrove, E. A., Caldon, C. E., Barraclough, J., Stone, A., and Sutherland, R. L. (2011). Cyclin D as a Therapeutic Target in Cancer. Nat. Rev. Cancer 11, 558-572. doi: $10.1038 / \mathrm{nrc} 3090$

Nakajima, S., Lan, L., Wei, L., Hsieh, C. L., Rapic-Otrin, V., Yasui, A., et al. (2014). Ubiquitin-specific Protease 5 Is Required for the Efficient Repair of DNA Double-Strand Breaks. PLoS One 9, e84899. doi:10.1371/journal.pone.0084899

Okada, K., Ye, Y. Q., Taniguchi, K., Yoshida, A., Akiyama, T., Yoshioka, Y., et al. (2013). Vialinin A Is a Ubiquitin-specific Peptidase Inhibitor. Bioorg. Med. Chem. Lett. 23, 4328-4331. doi:10.1016/j.bmcl.2013.05.093

Ostrom, Q. T., Gittleman, H., Liao, P., Rouse, C., Chen, Y., Dowling, J., et al. (2014). CBTRUS Statistical Report: Primary Brain and central Nervous System Tumors Diagnosed in the United States in 2007-2011. Neuro-Oncology 16 (Suppl. 4), iv1-iv63. doi:10.1093/neuonc/nou223

Pal, A., Young, M. A., and Donato, N. J. (2014). Emerging Potential of Therapeutic Targeting of Ubiquitin-specific Proteases in the Treatment of Cancer. Cancer Res. 74, 4955-4966. doi:10.1158/0008-5472.can-14-1211

Panner, A., Crane, C. A., Weng, C., Feletti, A., Fang, S., Parsa, A. T., et al. (2010). Ubiquitin-specific Protease 8 Links the PTEN-Akt-AIP4 Pathway to the
Control of FLIPS Stability and TRAIL Sensitivity in Glioblastoma Multiforme. Cancer Res. 70, 5046-5053. doi:10.1158/0008-5472.can-09-3979

Qian, G., Ren, Y., Zuo, Y., Yuan, Y., Zhao, P., Wang, X., et al. (2016). Smurf1 Represses TNF- $\alpha$ Production through Ubiquitination and Destabilization of USP5. Biochem. Biophysical Res. Commun. 474, 491-496. doi:10.1016/ j.bbrc.2016.04.135

Rape, M. (2018). Ubiquitylation at the Crossroads of Development and Disease. Nat. Rev. Mol. Cell Biol 19, 59-70. doi:10.1038/nrm.2017.83

Reardon, D. A., and Mitchell, D. A. (2017). The Development of Dendritic Cell Vaccine-Based Immunotherapies for Glioblastoma. Semin. Immunopathol 39, 225-239. doi:10.1007/s00281-016-0616-7

Rowinsky, E. K., Paner, A., Berdeja, J. G., Paba-Prada, C., Venugopal, P., Porkka, K., et al. (2020). Phase 1 Study of the Protein Deubiquitinase Inhibitor VLX1570 in Patients with Relapsed And/or Refractory Multiple Myeloma. Invest. New Drugs 38, 1448-1453. doi:10.1007/s10637-020-00915-4

Santoni, M., Burattini, L., Nabissi, M., Beatrice Morelli, M., Berardi, R., Santoni, G., et al. (2013). Essential Role of Gli Proteins in Glioblastoma Multiforme. Cpps 14, 133-140. doi:10.2174/1389203711314020005

Siebzehnrubl, F. A., Silver, D. J., Tugertimur, B., Deleyrolle, L. P., Siebzehnrubl, D., Sarkisian, M. R., et al. (2013). The ZEB1 Pathway Links Glioblastoma Initiation, Invasion and Chemoresistance. EMBO Mol. Med. 5, 1196-1212. doi:10.1002/ emmm.201302827

Stemkowski, P., García-Caballero, A., De Maria Gadotti, V., M’Dahoma, S., Huang, S., Gertrud Black, S. A., et al. (2017). TRPV1 Nociceptor Activity Initiates USP5/T-type Channel-Mediated Plasticity. Cell Rep. 18, 2289-2290. doi:10.1016/j.celrep.2017.02.050

Stupp, R., Hegi, M. E., Mason, W. P., van den Bent, M. J., Taphoorn, M. J., Janzer, R. C., et al. (2009). Effects of Radiotherapy with Concomitant and Adjuvant Temozolomide versus Radiotherapy Alone on Survival in Glioblastoma in a Randomised Phase III Study: 5-year Analysis of the EORTC-NCIC Trial. Lancet Oncol. 10, 459-466. doi:10.1016/s1470-2045(09)70025-7

Sun, H.-L., Zhu, A. C., Gao, Y., Terajima, H., Fei, Q., Liu, S., et al. (2020). Stabilization of ERK-Phosphorylated METTL3 by USP5 Increases m6A Methylation. Mol. Cell 80, 633-647. doi:10.1016/j.molcel.2020.10.026

Swatek, K. N., and Komander, D. (2016). Ubiquitin Modifications. Cell Res 26, 399-422. doi:10.1038/cr.2016.39

Turnbull, A. P., Ioannidis, S., Krajewski, W. W., Pinto-Fernandez, A., Heride, C., Martin, A. C. L., et al. (2017). Molecular Basis of USP7 Inhibition by Selective Small-Molecule Inhibitors. Nature 550, 481-486. doi:10.1038/nature24451

Vashistha, V., Bhardwaj, S., Yadav, B. K., and Yadav, A. K. (2020). Depleting Deubiquitinating Enzymes Promotes Apoptosis in Glioma Cell Line via RNA Binding Proteins SF2/ASF1. Biochem. Biophys. Rep. 24, 100846. doi:10.1016/ j.bbrep.2020.100846

Wang, J., Wakeman, T. P., Lathia, J. D., Hjelmeland, A. B., Wang, X. F., White, R. R., et al. (2010). Notch Promotes Radioresistance of Glioma Stem Cells. Stem Cells 28, 17-28. doi:10.1002/stem.261

Wang, S., Juan, J., Zhang, Z., Du, Y., Xu, Y., Tong, J., et al. (2017). Inhibition of the Deubiquitinase USP5 Leads to C-Maf Protein Degradation and Myeloma Cell Apoptosis. Cell Death Dis 8, e3058. doi:10.1038/cddis.2017.450

Wang, X., D’Arcy, P., Caulfield, T. R., Paulus, A., Chitta, K., Mohanty, C., et al. (2015). Synthesis and Evaluation of Derivatives of the Proteasome Deubiquitinase Inhibitor B-AP15. Chem. Biol. Drug Des. 86, 1036-1048. doi:10.1111/cbdd.12571

Weisberg, E. L., Schauer, N. J., Yang, J., Lamberto, I., Doherty, L., Bhatt, S., et al. (2017). Inhibition of USP10 Induces Degradation of Oncogenic FLT3. Nat. Chem. Biol. 13, 1207-1215. doi:10.1038/nchembio.2486

Weller, M., Wick, W., Aldape, K., Brada, M., Berger, M., Pfister, S. M., et al. (2015). Glioma. Nat. Rev. Dis. Primers 1, 15017. doi:10.1038/nrdp.2015.17

Wilkinson, K. D., Tashayev, V. L., O'Connor, L. B., Larsen, C. N., Kasperek, E., and Pickart, C. M. (1995). Metabolism of the Polyubiquitin Degradation Signal: Structure, Mechanism, and Role of Isopeptidase T. Biochemistry 34, 14535-14546. doi:10.1021/bi00044a032

Wu, H. C., Lin, Y. C., Liu, C. H., Chung, H. C., Wang, Y. T., Lin, Y. W., et al. (2014). USP11 Regulates PML Stability to Control Notch-Induced Malignancy in Brain Tumours. Nat. Commun. 5, 3214. doi:10.1038/ncomms4214

Wu, L., Zhang, C., Chu, M., Fan, Y., Wei, L., Li, Z., et al. (2020). miR-125a Suppresses Malignancy of Multiple Myeloma by Reducing the Deubiquitinase USP5. J. Cell Biochem 121, 642-650. doi:10.1002/jcb.29309 
Xia, Q., Zhang, H., Zhang, P., Li, Y., Xu, M., Li, X., et al. (2020). Oncogenic Smurf1 Promotes PTEN Wild-type Glioblastoma Growth by Mediating PTEN Ubiquitylation. Oncogene 39, 5902-5915. doi:10.1038/ s41388-020-01400-1

Xie, X., Matsumoto, S., Endo, A., Fukushima, T., Kawahara, H., Saeki, Y., et al. (2018). Deubiquitylases USP5 and USP13 Are Recruited to and Regulate HeatInduced Stress Granules through Their Deubiquitylating Activities. J. Cell Sci 131, 1. doi:10.1242/jcs.210856

Xu, X., Huang, A., Cui, X., Han, K., Hou, X., Wang, Q., et al. (2019). Ubiquitin Specific Peptidase 5 Regulates Colorectal Cancer Cell Growth by Stabilizing Tu Translation Elongation Factor. Theranostics 9, 4208-4220. doi:10.7150/ thno.33803

Xue, S., Wu, W., Wang, Z., Lu, G., Sun, J., Jin, X., et al. (2020). USP5 Promotes Metastasis in Non-small Cell Lung Cancer by Inducing EpithelialMesenchymal Transition via Wnt/beta-Catenin Pathway. Front. Pharmacol. 11, 668. doi:10.3389/fphar.2020.00948

Yan, F.-J., Zhang, X.-J., Wang, W.-X., Ji, Y.-X., Wang, P.-X., Yang, Y., et al. (2017). The E3 Ligase Tripartite Motif 8 Targets TAK1 to Promote Insulin Resistance and Steatohepatitis. Hepatology 65, 1492-1511. doi:10.1002/hep.28971

Young, M. J., Hsu, K. C., Lin, T. E., Chang, W. C., and Hung, J. J. (2019). The Role of Ubiquitin-specific Peptidases in Cancer Progression. J. Biomed. Sci. 26, 42. doi:10.1186/s12929-019-0522-0
Yuan, J., Li, X., Zhang, G., Cheng, W., Wang, W., Lei, Y., et al. (2021). USP39 Mediates P21-dependent Proliferation and Neoplasia of colon Cancer Cells by Regulating the p53/p21/CDC2/cyclin B1 axis. Mol. Carcinogenesis 60, 265-278. doi: $10.1002 / \mathrm{mc} .23290$

Conflict of Interest: The authors declare that the research was conducted in the absence of any commercial or financial relationships that could be construed as a potential conflict of interest.

Publisher's Note: All claims expressed in this article are solely those of the authors and do not necessarily represent those of their affiliated organizations, or those of the publisher, the editors and the reviewers. Any product that may be evaluated in this article, or claim that may be made by its manufacturer, is not guaranteed or endorsed by the publisher.

Copyright (C) $2021 \mathrm{Li}$, Yang, Chen, Bao, Wu, Hu, Feng, Xu, Li, Li, Jin, Xu, Zhang, Qian and Pan. This is an open-access article distributed under the terms of the Creative Commons Attribution License (CC BY). The use, distribution or reproduction in other forums is permitted, provided the original author(s) and the copyright owner(s) are credited and that the original publication in this journal is cited, in accordance with accepted academic practice. No use, distribution or reproduction is permitted which does not comply with these terms. 